\title{
Angelica glauca root extract mediated synthesis of silver nanoparticles and their investigations for biological and electrical applications
}

\author{
Madhulika Bhagat ${ }^{1,}$, Sandeep Arya ${ }^{2}$ and Shayana Rajput ${ }^{1}$ \\ ${ }^{1}$ School of Biotechnology ${ }^{2}$ Department of Physics \\ University of Jammu, Jammu-180006, India \\ *Email: madhulikasbt@gmail.com
}

In our present work Angelica glauca, a plant grown in high altitudes of Jammu and Kashmir was used to synthesize silver nanoparticles. $\mathrm{AgNO}_{3}$ was reduced by using plant extract as shown in Figure 1. These nanoparticles were further characterized and used to study their biological and electrical properties. The characterization was done using techniques such as: UV-Vis spectroscopy, SEM, X-Ray, EDX etc. After characterization, antioxidant potential of these nanoparticels was carried on by using DPPH and Lipid perioxidation inhibition assay. It was analysed that the antioxidant potential of these synthesized nano particles was higher than the plant extract.
Electron microscopy is used to characterize the shape and size of synthesised nanoparticles. SEM is a type of electron microscope that images a sample by scanning it with a highenergy beam of electrons. After the preparation of the nanoparticles, the suspension of nanoparticles in double distilled water was used for SEM analysis using Zeiss EVO 40 Scanning Electron Microscope by fabricating a drop of suspension onto a clean electric Stubs and allowing water to completely evaporate. The dried sample was then mounted on a clean aluminium stub. The sample was then viewed under scanning electron microscope at an accelerating voltage of $20 \mathrm{kV}$.

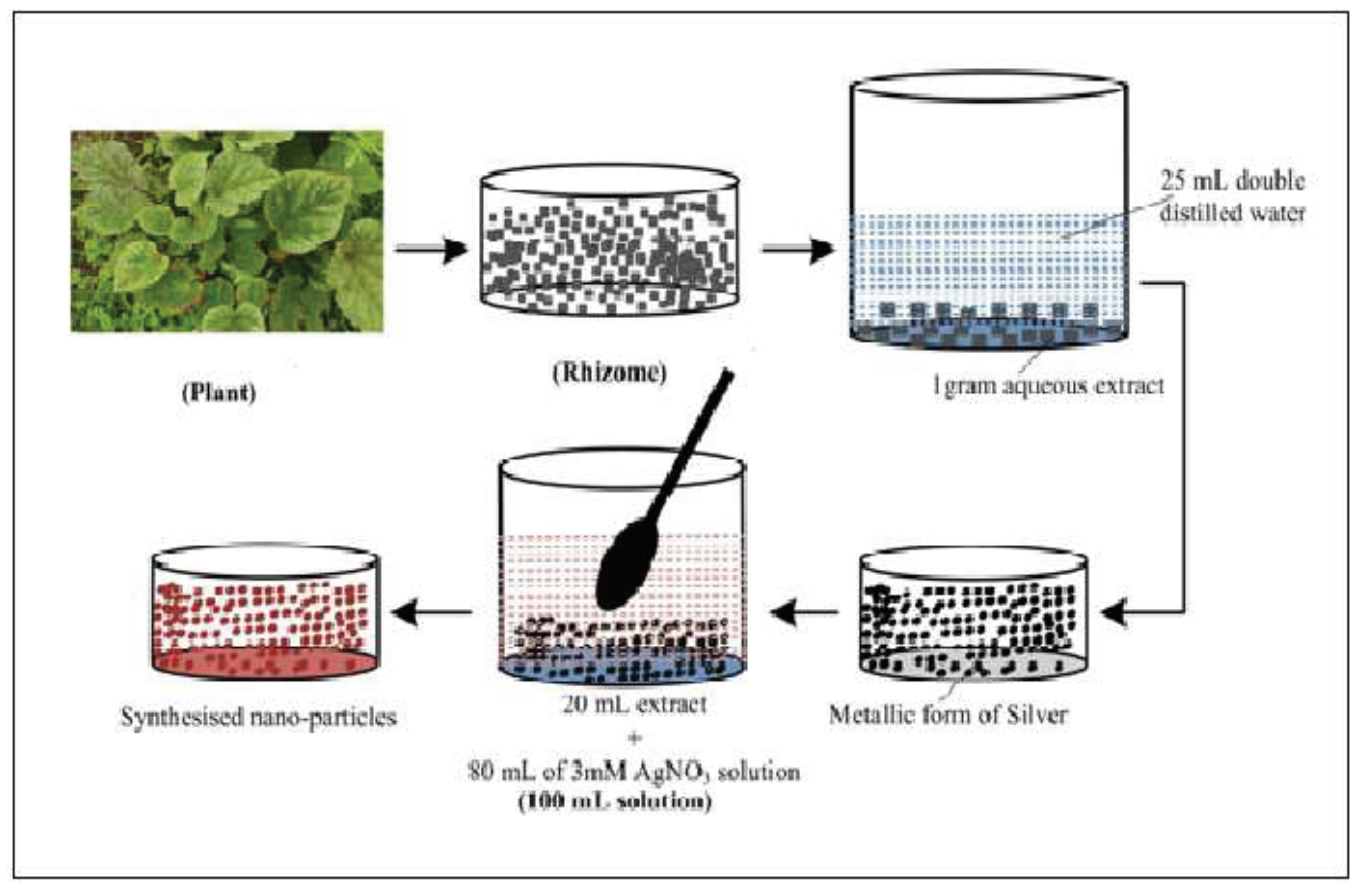

Figure 1: Diagrammatic representation of synthesis of plant mediated silver nanoparticles 